\title{
Academic hospital accreditation strengthens postgraduate training programmes: Case study from Universitas Academic Hospital
}

\author{
A Linegar, S Whittaker, G van Zyl
}

Benefits derived from the Council for Health Services Accreditation South Africa (COHSASA) accreditation of the Universitas Academic Hospital (UAH) in Bloemfontein are illustrated. Accreditation assessments were performed between 2001 and 2007, and full compliance with the COHSASA standards for Academic Hospitals was achieved. An initiative to develop thoracic surgery in central South Africa (SA) was launched by the Department of Cardiothoracic Surgery at UAH. The synergistic effects of quality improvements in healthcare provision owing to the accreditation process, and the project to increase service provision in thoracic surgery in central SA, have led to a qualitative and quantitative increase in thoracic surgical service provision. The importance of academic hospital accreditation in strengthening postgraduate training programmes is shown, and the accreditation process is recommended for all South African academic teaching hospitals to support, improve and sustain our training platforms.

S Afr Med J 2012;102:146-148.
This case study originated from the combined experiences of the accreditation process of a major teaching hospital in central South Africa (SA) and a simultaneous development initiative in general thoracic surgery in the region.

The Council for Health Services Accreditation South Africa (COHSASA) performed serial accreditation assessments between 2001 and 2007 at the Universitas Academic Hospital (UAH),

Department of Cardiothoracic Surgery, University of the Free State, Bloemfontein A Linegar, MB ChB, FCS SA (Cardio), $\mathrm{PhD}$

Council for Health Services Accreditation South Africa (COHSASA), University of Pretoria

S Whittaker, $\mathrm{MB} \mathrm{ChB}$, Med (CH), FCM (CH), MD

Faculty of Health Sciences, University of the Free State G van Zyl, MB ChB, MFamMed, MBA, PhD
Bloemfontein, a tertiary-level referral and training hospital for central SA's population of approximately 5 million people. Concurrently, an initiative to develop thoracic surgery in central SA was launched by the Department of Cardiothoracic Surgery at UAH. ${ }^{1}$

We describe the influence of hospital accreditation on postgraduate training. The objectives were: (i) explanation of the accreditation process; (ii) the rationale for improving postgraduate training; (iii) the relationship between clinical activity and academic productivity; and (iv) the benefits of hospital accreditation and quality management. The study illustrates the positive influence of the accreditation process on the quality of clinical service delivery and, in consequence, on postgraduate training standards.

\section{Rationale for improving postgraduate training in SA}

Tertiary healthcare is in a crisis after nearly 3 decades of neglect, ${ }^{2,3}$ compounded by the shortage of doctors and specialists in SA. An analysis commissioned by the Hospital Association of SA (HASA) estimated the number of doctors in the country to be 27431 , of whom 17802 are general practitioners and 9630 are specialists. ${ }^{4,5}$ These figures are probably the most accurate estimate currently available. 
The current 55 doctors per 100000 population compares poorly with rates in the developed world. The UK and the USA report 230 and 256 doctors per 100000 population, respectively. SA also falls short of comparable countries of a similar economic setting, such as Brazil, that has 185 doctors per 100000 population. These comparisons indicate the SA shortage to be approximately 65000 doctors. ${ }^{4}$ It is also important to note that the specialist population is ageing, with $38 \%$ in state employ being over the age of 50 years.

The SA public sector is estimated to require 15 specialists per 100 000 population $-\mathrm{v}$. the current 7 specialists per 100000 population. To meet the target requirement in 15 years, current registrar admissions would have to increase by $58 \%$ (personal communication, Professor Wynand Van Der Merwe, 2008).

A most serious problem facing the future of postgraduate training is the high level of vacancy rates in specialist and registrar posts in state sector healthcare. The figure varies by province, from as high as $80 \%$ vacancy in Limpopo to $42 \%$ for the Free State and $22 \%$ for Gauteng. The shortfall in specialists results in a leadership deficiency in the short term, which inevitably becomes an institutional inability to train doctors in the long term. The situation is especially serious in specialised fields such as thoracic surgery, where the loss of one specialist can be critical to the sustainability of a service and a training platform. Activities such as outreach programmes, which have high human resource requirements, are similarly affected.

While critically undermining the ability to deliver a clinical service and train doctors at all levels, such staff shortages also reduce the capacity for relevant clinical research. The reluctance to fill posts exacerbates staff losses as current incumbents find it increasingly difficult to achieve their academic goals in an unsatisfactory working environment.

\section{Addressing the problem - the UAH experience}

Hospital accreditation is a systematic external audit of the administrative, managerial, clinical and patient safety aspects of a hospital. It is a tool employed by healthcare organisations to quantify the performance of healthcare facilities relative in terms of a set of standards, with the objective of quality assurance and quality improvement in service delivery.

The management of UAH decided in 2001 to enter the hospital in the COHSASA accreditation programme, which was subsequently confirmed by formal processes in 2004 . The COHSASA standards define functions, structures and processes that are required for clinical departments to provide safe quality services to patients. Since the COHSASA programme required an initial baseline assessment against the standards, it was felt that this assessment would assist to identify significant deficiencies in the work environment that militated against safe quality service provision. ${ }^{6}$

The evaluation process begins with the qualitative and quantitative assessment of several pre-determined criteria. Hospitals are categorised as non-compliant, partially compliant or compliant with each criterion relative to its related standard. Full compliance in a specific criterion would score 100 . Non-compliant and partially compliant criteria are further categorised, according to their clinical importance, as being mildly, moderately or seriously non- or partially compliant.

The overall hospital score is then calculated from the weighted criterion scores, which places a hospital into one of four score categories, viz. weak (0 - 40), poor (40 - 80), acceptable (80 - 90) and $\operatorname{good}(90-100)$.
To be fully accredited, a hospital should substantially comply with all standards, meaning that all services should score at least $80 / 100$. There should be no areas of non-compliance in standards that potentially compromise patient or staff safety or contravene health regulations. Furthermore, there should be no serious administrational, organisational or managerial problems.

Facilities that do not achieve accreditation at their first assessment are categorised as being in the entry level grade if they score between 60 and 70 on overall compliance, or intermediate level grade if they score between 70 and 85 on overall compliance. These preaccreditation grades are useful in providing support for a facility that can be fully accredited once the identified problem areas are attended to.

UAH was not initially fully accredited. After addressing the problems identified in the 2001 accreditation survey, full accreditation was awarded in 2004 and 2007.

Fig. 1 shows that the UAH has been able to retain these high levels of standards compliance and has provided a safe quality environment in which the multidisciplinary team has been able to provide quality care for its patients. The maintenance of standards has also demonstrated that evidence-based medicine is practised in the facility.

\section{Relationship between clinical activity and academic productivity}

During the period 2004 - 2010, a project aimed at the development of thoracic surgery in central SA was initiated at $\mathrm{UAH} .{ }^{1}$ This team project achieved significant developments in clinical service delivery, academic training and clinical research in thoracic surgery. It ran concurrently with the overall hospital developments under the COHSASA accreditation process and provided an excellent example of how the accreditation process feeds into postgraduate training programmes.

Part of the thoracic surgery development project focused on the measurement of burden of disease, clinical activity and academic productivity, which in this context includes doctor training. Fig. 2 is a conceptual diagram that illustrates the relationship between these elements; it demonstrates that all healthcare activities occur within a specific healthcare policy and governance context and, furthermore, that clinical activity represents a measurable portion of the burden of disease in the community. The difference between the actual clinical service provided and the expected provision based on the burden of disease is the performance gap. Academic productivity exists within

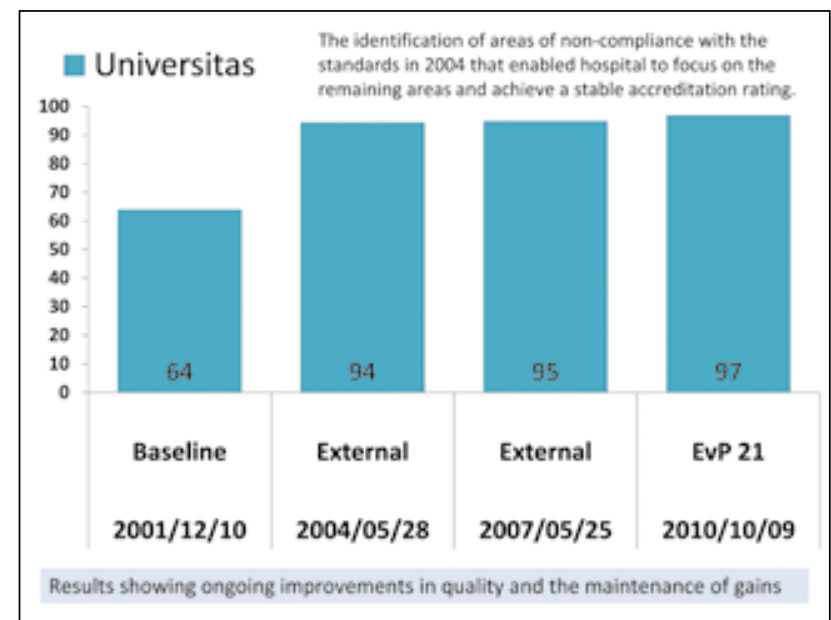

Fig. 1. Overall standard compliance scores achieved in follow-up surveys for the period $2004-2010$. 


\section{Original Articles}

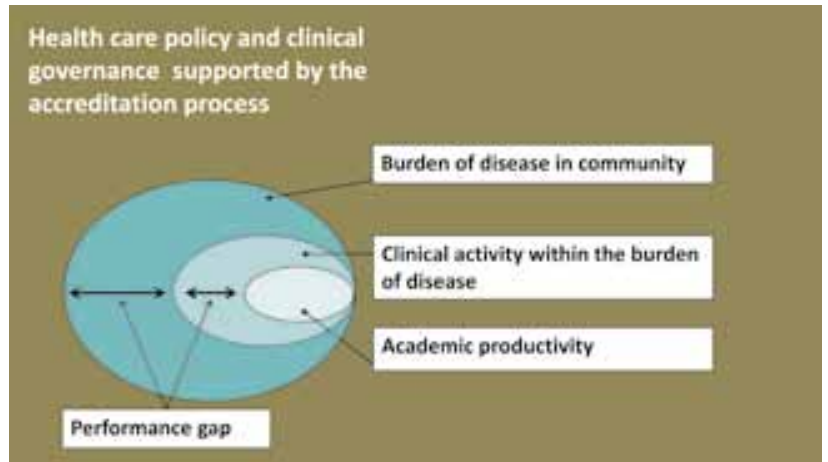

Fig. 2. The relationship between burden of disease, clinical activity and academic productivity. ${ }^{1}$

the available clinical workload and seeks to answer research questions originating from clinical uncertainties. Without a critical mass of clinical activity, training and research are not possible.

Link between the accreditation process and improvements in clinical service delivery

The beneficial effects of the accreditation process upon clinical service delivery were apparent in this retrospective case study. The results of the serial assessments of the UAH since 2001 and their concurrence with the thoracic surgery development project have enabled the identification of several benefits, as follows. The process:

- is able to identify problem areas and constraints in service provision and areas of excellence

- promotes improvements in the administrative and managerial functions of the hospital that underpin the ability to provide efficient, caring healthcare

- provides a platform for quality assurance in healthcare

- encourages continual improvement in healthcare standards

- forms a basis of information and knowledge on which attainable and appropriate healthcare standards can be constructed
- creates a culture of accountability, in both the internal and external environments of the hospital

- forms a basis for advocacy to local and national healthcare governance

- provides a sound basis for quality tertiary care, which is one of the important pillars of the future establishment of a national health insurance scheme.

\section{Conclusion}

The COHSASA experience at UAH confirms that many favourable changes are brought about by the accreditation process. The repeated assessments at predetermined intervals have also shown the improvements to be important and sustainable. While a facility may not achieve full accreditation at its first evaluation, it is the process of correcting identified deficiencies that brings about the important changes in the organisation. These changes concern the development of a culture of quality improvement in service delivery at all levels of the healthcare facility. Quality improvement in service delivery to patients and the community, and in the working conditions of the healthcare providers, is the ultimate goal of the accreditation process.

The experience of this case study is supportive of the accreditation process for all academic teaching hospitals in SA in the interests of supporting, improving and sustaining our training platforms.

\section{References}

1. Linegar AG. A model for the development of thoracic surgery in central South Africa. PhD thesis, University of the Free State, 2008

. Kirsch R. A model for the governance of academic health centres. Transactions 2009;53(1):34-37.

3. Gevers W. Clinical research in South Africa: a core asset under pressure. Lancet 2009;374:760-762.

4. Econex. Updated GP and Specialist Numbers for SA. Health Reform Note 7. http://www.econex.co za/ images/stories/ECONEX_Health\%20Reform\%20Note_7.pdf (accessed 8 December 2010).

5. Econex. The Human Resource Supply Constraint: The Case of Doctor. Health Reform Note 8 . http://www.econex.co.za/images/stories/ECONEX_Health\%20Reform\%20Note_8.pdf (accessed 8 http://www.econex

6. Whittaker S, Green-Thompson R, McCusker I, Neyembezi B. Status of a health care quality review programme in South Africa. Int J Qual Health Care 2000;12:247-250.

Accepted 19 August 2011. 